\title{
STUDY OF POLYMORPHISM OF BOROVANADATE GLASS OF SODIUM BY RAMAN SPECTROSCOPY LOW FREQUENCIES
}

\author{
M. K. Rabia ${ }^{*}$, M. Mayoufi, L. Grosvalet and B. Champagnon
}

Université Badji Mokhtar - Annaba; Laboratoire de Physique des Rayonnements, Département de Physique, B.P. 12, Annaba, 23000, Algérie.

\begin{abstract}
Sodium tetraborate $(100-\mathrm{x})\left(\mathrm{Na}_{2} \mathrm{~B}_{4} \mathrm{O}_{7} \cdot 10 \mathrm{H}_{2} \mathrm{O}\right)-\mathrm{xV}_{2} \mathrm{O}_{5},(\mathrm{x}=0$ to 20 mole $\%)$ has been elaborated by splat cooling technique. Raman Measurements on the doped and non polish samples reveal the presence of the of $\alpha-\mathrm{NaVO}_{3}$ crystal on the superficial layer. After polishing, Raman spectra characteristic of glasses are obtained with two main bands located at 555 and $1097 \mathrm{~cm}^{-1}$ in the undoped glass and four bands at 241, 381, 776 and $938 \mathrm{~cm}^{-1}$ for the vanadium oxyde doped glasses. The volume devitrification of these glasses occurs at $750^{\circ} \mathrm{C}$ and the $\beta$-NaVO3 crystalline phase is identified by Raman scattering.
\end{abstract}

Keywords: Oxide glasses, Non-linear optics, Raman scattering, XRD, DSC.

\section{INTRODUCTION}

Les verres de borovanadate de sodium sont une catégorie particulière contenant deux verres formateurs, $\mathrm{B}_{2} \mathrm{O}_{3}$ et $\mathrm{V}_{2} \mathrm{O}_{5}$. Lorsqu'un alcalin est ajouté, la connectivité et les nombres de coordination dans le verre peuvent varier de différente manière. À notre connaissance, le processus de dévitrification de ces verres n'a pas été étudié jusqu'à maintenant bien que certains cristaux tels que $\mathrm{NaVO}_{3}$ sont des minéraux naturels (munirite $\mathrm{NaVO}_{3} \cdot 2 \mathrm{H}_{2} \mathrm{O}$ ) largement utilisés dans l'industrie et la pharmacologie [1].

Author Correspondence, e-mail: mkamel.rabia@gmail.com

ICID: 1020740 
La phase la plus commune, $\alpha-\mathrm{NaVO}_{3}$, appartient à la famille des pyroxènes. Glazyrin [2] and Sørum [3] montrèrent que le cristal de $\alpha-\mathrm{NaVO}_{3}$ est monoclinique avec $\mathrm{Z}=8$. La phase $\beta-\mathrm{NaVO}_{3}$ est orthorhombique. La phase cristalline, $\beta-\mathrm{NaVO}_{3}$, préparée à partir de $\mathrm{NaOH}$ et $\mathrm{NH}_{4} \mathrm{VO}_{3}$ est stable de la température ambiante jusqu'à $405^{\circ} \mathrm{C}$ puis se transforme irréversiblement en $\alpha-\mathrm{NaVO}_{3}$ [4].

Le verre de borovanadate de sodium a été étudié par Muthupari [5]. Dans cet article, des verres $\mathrm{Na}_{2} \mathrm{~B}_{4} \mathrm{O}_{7}$ contenant jusqu'à $20 \%$ de $\mathrm{V}_{2} \mathrm{O}_{5}$ ont été élaborés. La structure des verres et leurs cristallisations sous recuit thermique sont analysées par spectroscopie Raman et diffraction X (XRD).

\section{2. ÉTUDE EXPERIMENTALE}

\section{Élaboration des verres}

De la poudre $\mathrm{Na}_{2} \mathrm{~B}_{4} \mathrm{O}_{7} .10 \mathrm{H}_{2} \mathrm{O}(\% \mathrm{~mol})$ contenant différentes quantités de $\mathrm{V}_{2} \mathrm{O}_{5}$ entre 0 et $20 \%$ ont été chauffés à $1000^{\circ} \mathrm{C}$ pendant 1 heure. La masse fondue est refroidie rapidement dans un moule en acier inoxydable à la température ambiante ou bien le liquide est écrasé entre deux rouleaux métalliques tournant à grande vitesse l'un contre l'autre [Tableau 1]. Les échantillons de verres ont des épaisseurs n'excédant pas $2 \mathrm{~mm}$ et sont étudiés avant et après polissage de leurs surfaces.

\section{Dispositif expérimental}

Les spectres micro-Raman ont été enregistrés avec un spectrophotomètre Raman Renishaw RM 1000 de $100 \mathrm{~mW}$ utilisant une raie laser argon à $514 \mathrm{~nm}$. Les spectres sont obtenus grâce à l'objectif d'un microscope optique qui reçoit également le faisceau rétrodiffusé dans son axe optique à travers un objectif de grossissement x50. Les spectres non polarisés ont été recueillis à température ambiante entre $100 \mathrm{~cm}^{-1}$ et $1100 \mathrm{~cm}^{-1}$ pour l'échantillon non dopé et pour les échantillons contenant jusqu'à $20 \%$ (mol.) de $\mathrm{V}_{2} \mathrm{O}_{5}$ (Tableau 1). 
Tableau 1. Échantillons de verres de borovanadate de sodium, dopés à l'oxyde de vanadium avec des concentrations croissantes, en accord avec la formule molaire (X \%mol.) $\mathrm{Na}_{2} \mathrm{~B}_{4} \mathrm{O}_{7} .10 \mathrm{H}_{2} \mathrm{O}-\left((100-\mathrm{X}) \%\right.$ mol.) $\mathrm{V}_{2} \mathrm{O}_{5}$.

\begin{tabular}{|c|c|c|}
\hline Échantillons & $\begin{array}{c}\mathrm{Na}_{2} \mathrm{~B}_{4} \mathrm{O}_{7} \cdot 10 \mathrm{H}_{2} \mathrm{O} \\
{[\% \mathrm{~mol}]}\end{array}$ & $\begin{array}{c}\mathrm{V}_{2} \mathrm{O}_{5} \\
{[\% \mathrm{~mol}]}\end{array}$ \\
\hline 1 & 100 & 0 \\
\hline 2 & 95 & 5 \\
\hline 3 & 92.5 & 7.5 \\
\hline 4 & 90 & 10 \\
\hline 5 & 87.5 & 12.5 \\
\hline 6 & 85 & 15 \\
\hline 7 & 80 & 20 \\
\hline
\end{tabular}

\section{RESULTATS}

La cristallisation de surface a été soigneusement éliminée par polissage des échantillons qui leur confère une bonne qualité optique. Les spectres Raman des différents échantillons après polissage sont présentés sur la Fig. 1. L'échantillon non dopé (Fig. 1 (a)) est dominé par deux raies à $555 \mathrm{~cm}^{-1}$ et $1097 \mathrm{~cm}^{-1}$. Les échantillons dopés au vanadium sont caractérisés par une bande principale à $938 \mathrm{~cm}^{-1}$.

Avant le polissage, les échantillons de 2 à 7 montrent des raies fines intenses, à $956 \mathrm{~cm}^{-1}$ et $509 \mathrm{~cm}^{-1}$ comme, par exemple, pour l'échantillon 7 sur la Fig. 2 (a).

Le recuit thermique sous atmosphère, pour $210 \mathrm{mn}$ jusqu'à $700^{\circ} \mathrm{C}$, de l'échantillon 7 à $20 \%$ de $\mathrm{V}_{2} \mathrm{O}_{5}$, ne change pas le spectre Raman. Un traitement thermique à $750{ }^{\circ} \mathrm{C}(210$ $\mathrm{mn})$ suivie d'un refroidissement lent donne un spectre Raman intense avec des raies fines (Fig. 4 (a)). Les spectres XRD de la poudre préparée à partir des échantillons traités thermiquement montrent des raies fines correspondant à la fois aux $\mathrm{NaVO}_{3} \cdot 2 \mathrm{H}_{2} \mathrm{O}$ et aux cristaux $\mathrm{Na}_{2} \mathrm{~B}_{4} \mathrm{O}_{7} .5 \mathrm{H}_{2} \mathrm{O}$ superposée sur de larges bandes (Fig. 5).

\section{INTERPRETATION}

Le spectre Raman de l'échantillon non dopé (Fig. 1 (a)) correspond à celui d'un matériau amorphe. Les principales bandes correspondent à $555 \mathrm{~cm}^{-1}$ et $1097 \mathrm{~cm}^{-1}$. Les cristaux à la surface des échantillons 2 à 7 peuvent être identifiés comme étant $\alpha$ $\mathrm{NAVO}_{3}$ [9] (fig. 2). La raie principale à $956 \mathrm{~cm}^{-1}$ est attribuée à l'étirement des modes 
symétriques de $\mathrm{VO}_{2}$. Après polissage, les raies cristallines sont éliminées et les bandes larges Raman (Fig. 1) des échantillons dopés sont caractéristiques d'un verre.

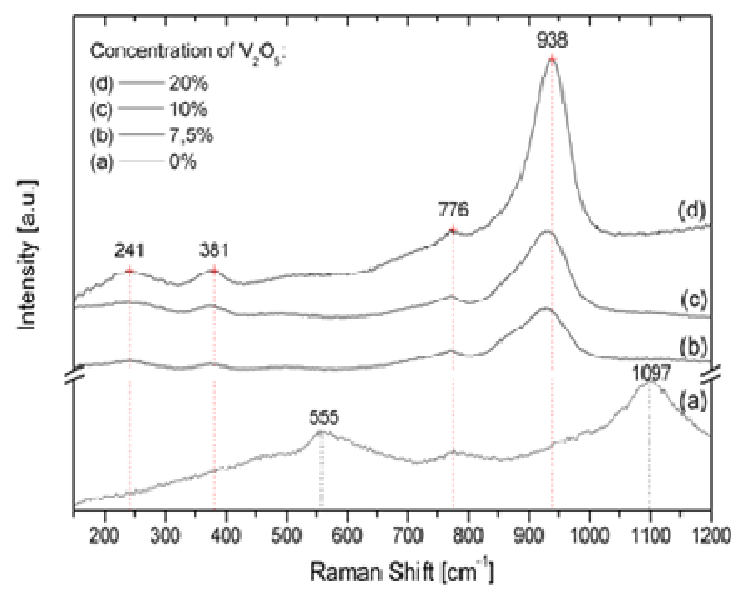

Fig.1. Spectres Raman des échantillons de tétraborate de sodium $\left(\mathrm{Na}_{2} \mathrm{~B}_{4} \mathrm{O}_{7} \cdot 10 \mathrm{H}_{2} \mathrm{O}\right)$ avec différentes concentrations de $\mathrm{V}_{2} \mathrm{O}_{5}$.

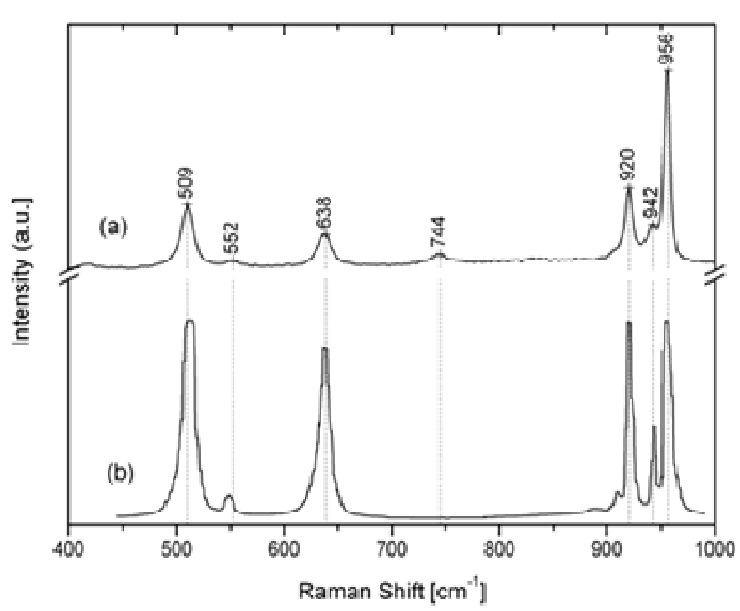

Fig.2. Comparaison du spectre Raman de l'échantillon 7(a) avant polissage avec celui de (b) du cristal de $\alpha-\mathrm{NaVO}_{3}$ [9].

Les spectres Raman des verres de plus de 7,5\% de $\mathrm{V}_{2} \mathrm{O}_{5}$ sont dominés par une large bande liée aux vibrations $\mathrm{V}=\mathrm{O}$. Cette bande se déplace de $917 \mathrm{~cm}^{-1}$ à $931 \mathrm{~cm}^{-1}$ dans les verres $(100-\mathrm{x})\left[0,33 \mathrm{~B}_{2} \mathrm{O}_{3}-0,67 \mathrm{ZnO}\right]-\mathrm{xV}_{2} \mathrm{O}_{5}$ pour $\mathrm{x}$ variant de 5 à 20 [10]. Les verres borovanadate de Lithium [11] montrent un spectre très similaire avec les principales vibrations $\mathrm{V}=0$ variant entre 890 et $950 \mathrm{~cm}^{-1}$ lorsque $\mathrm{V}_{2} \mathrm{O}_{5}$ varie jusqu'à $30 \%$ (mol.). Ceci est cohérent avec l'attribution des spectres Raman des verres borovanadate de sodium dopés au $\mathrm{V}_{2} \mathrm{O}_{5}$, dont la structure a été décrite à partir des études infrarouges et thermiques [5].

La stabilité de l'échantillon 7 est conservée pour les traitements thermiques au-dessus de Tg jusqu'à $700^{\circ} \mathrm{C}$. Cependant à $750^{\circ} \mathrm{C}$, après $210 \mathrm{mn}$, la cristallisation se produit. Le spectre Raman (Fig. 4) correspond à la phase cristalline $\beta-\mathrm{NaVO}_{3}$ [9] qui est composé de chaînes métavanadates doubles, les atomes $\mathrm{V}$ étant entouré par cinq atomes $\mathrm{O}$ pour former une bipyramide trigonale déformée [6]. L'analyse par XRD de cet échantillon traité thermiquement révèle que les deux espèces $\mathrm{NaVO}_{3} .2 \mathrm{H}_{2} \mathrm{O}$ et $\mathrm{Na}_{2} \mathrm{~B}_{4} \mathrm{O}_{7} .5 \mathrm{H}_{2} \mathrm{O}$ ont cristallisé bien qu'aucune raie correspondant aux cristaux $\mathrm{Na}_{2} \mathrm{~B}_{4} \mathrm{O}_{7}$ n'est observée dans les spectres Raman. 


\section{CONCLUSION}

Dans cet article, nous avons décrit les spectres Raman des verres borovanadate de sodium qui sont dominés par une bande très intense correspondant aux modes d'étirement de $\mathrm{VO}_{2}$ à $938 \mathrm{~cm}^{-1}$. La dévitrification se produit à $750^{\circ} \mathrm{C}$ et deux phases cristallines sont obtenues à partir de la diffraction X. La phase $\beta-\mathrm{NaVO} 3$ est identifié par diffusion Raman.

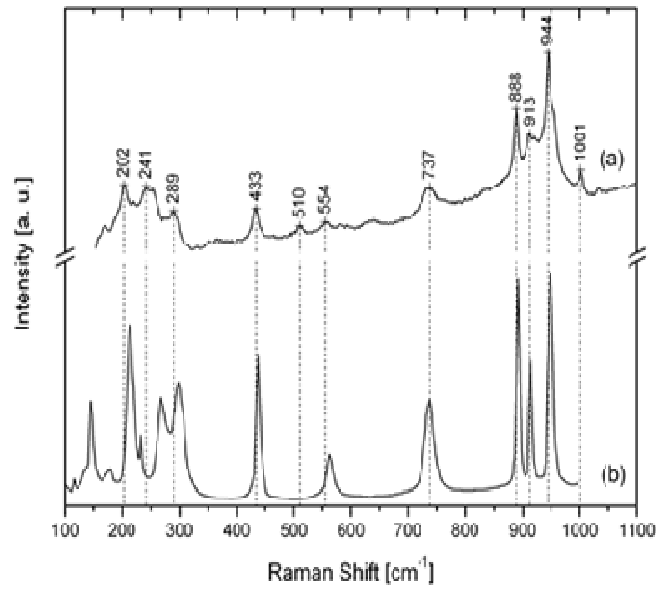

Fig.3. Spectres Raman du verre $\mathrm{Na}_{2} \mathrm{~B}_{4} \mathrm{O}_{7} \cdot 10 \mathrm{H}_{2} \mathrm{O}$, dopé à $20 \% \mathrm{~V}_{2} \mathrm{O}_{5}$ après recuit de $210 \mathrm{mn}$ à $750^{\circ} \mathrm{C}(\mathrm{a})$ et cristal de $\beta-\mathrm{NaVO}_{3}$ [Seetharaman [9]] (b).

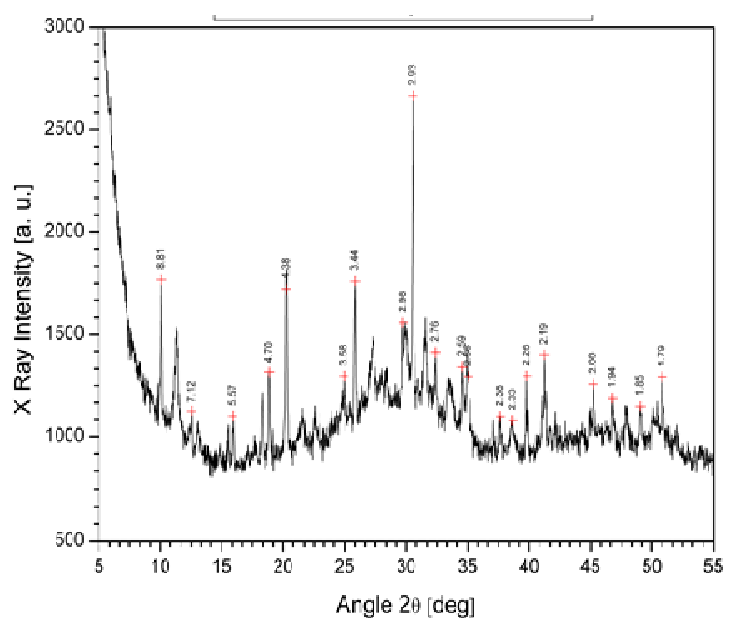

Fig.4. La série des raies indexées (les valeurs en $\AA$ ) de ce spectre de diffraction $\mathrm{X}$ correspond au $\mathrm{Na}_{2} \mathrm{~B}_{4} \mathrm{O}_{7} .5 \mathrm{H}_{2} \mathrm{O}$. et les autres (non indexeés) à $\mathrm{NaVO}_{3} \cdot 2 \mathrm{H}_{2} \mathrm{O}$.

\section{REFERENCES}

[1] Goldfine A. B., Simonson D. C., Folli F., Patti M. E. et Kahn C. R. Journal of Clinical Endocrinology \& Metabolism.1995, 80, 3311.

[2] Glazyrin M. P. Kristallografiya. 1965, 10, 761.

[3] Sørum H. et Videnskab K. N. Selskabs Forth. 1953, 16, 39.

[4] Luckacs I. et Strusievici C. Z. Anorg Allgem Chem. 1962, 315-323.

[5] Muthupari S., Prabakar S. et Rao K. J. J Phys Chem. 1994, 98, 2646-2652.

[6] Shen Z. X., Ong C. W., Tang S. H. et Kuok M. H. Physical Review B. 1994, 49(2), 1436. 
[7] Shen Z. X., Ong C. W., Tang S. H. et Kuok M. H. J. Phys Chem Solids. 1994, 55(8), 661-664.

[8] Devi S. A., Philip D. et Aruldhas G. J. Solid Stat Chem. 1994, 113, 157-162.

[9] Seetharaman S., Bhat H. L. et Narayanan P. S. J Raman Spectrosc. 1983, 14 (6), 401.

[10] Li H., Lin H., Chen W. et Luo L. Journal of Non-Cristalline Solids. 2006, 352, 3069-3073.

[11] Hübert T., Mosel G. et Witke K. Glass Physics and Chemistry. 2001, 27(2), $114-$ 120. 


\section{ÉTUDE DU POLYMORPHISME DU VERRE BOROVANADATE DE SODIUM PAR SPECTROSCOPIE RAMAN BASSES FREQUENCES}

\section{RESUME}

Le tétraborate de sodium de formule molaire $(100-\mathrm{x})(\mathrm{Na} 2 \mathrm{~B} 4 \mathrm{O} 7.10 \mathrm{H} 2 \mathrm{O})-\mathrm{xV} 2 \mathrm{O} 5$, ( $\mathrm{x}=0$ à $20 \%$ mole) a été élaboré par la technique "splat cooling " (technique marteau et enclume). Les mesures de spectres Raman réalisées sur des échantillons dopés, avant polissage, montrent tous une série de raies fines révélant la présence du cristal $\alpha$ $\mathrm{NaVO} 3$ qui s'est formé à leurs surfaces. Après polissage des échantillons, des spectres Raman caractéristiques des verres sont observés avec deux bandes principales situées à 555 et 1097 cm-1 sur des verres non dopés et quatre bandes à 241, 381, 776 et $938 \mathrm{~cm}-1$ sur des verres dopés avec l'oxyde de vanadium. La dévitrification en volume de ces verres apparait à $750^{\circ} \mathrm{C}$ et la phase cristalline $\beta-\mathrm{NaVO} 3$ est identifiée par diffusion Raman.

Mots-clés: Spectroscopie laser, Optique non linéaire, Verres oxyde, Diffusion Raman, XRD.

\section{How to cite this article}

Rabia M K, Mayoufi M, Grosvalet L and Champagnon B. Study of polymorphism of borovanadate glass of sodium by raman spectroscopy low frequencies. J Fundam Appl Sci. 2012, 4(1), 101-107. 